\title{
Quasi Swallowtail Singularities
}

Dedicated to the memory of Vladimir Zakalyukin

\author{
Fawaz Alharbi \\ Applied Science College/Department of Mathematical Sciences \\ Umm Alqura University \\ Makkah, Saudi Arabia \\ fdlohaibi@uqu.edu.sa
}

\begin{abstract}
We obtain a list of simple classes of singularities of function germs with respect to the quasi swallowtail equivalence relation. We discuss its connection with the singularities of Lagrangian projections in presence of a swallowtail. We also describe the bifurcation diagrams and caustics of simple quasi swallowtail singularities.
\end{abstract}

Keywords: Quasi border singularities, cusp, swallowtail, bifurcation diagram, caustic, Lagrangian projection.

\section{INTRODUCTION}

In 2007, Vladimir Zakalyukin classified function germs with respect to new non-standard equivalence relations (see $[1,2]$ ) which he named quasi equivalences. He was motivated by the needs of the theory of Lagrangian maps of singular varieties. In [2], the quasi relation is aimed to control positions of only critical points of functions defined on a space equipped with a smooth hypersurface and allow absolute freedom outside the critical locus. Later on and based on Zakalyukin's idea in $[3,4]$, more general settings were considered and similar equivalence relations were introduced for any hypersurface or a complete intersection which may be regular, singular or reducible . Such a hypersurface is called a border and the relation is called quasi border equivalence. In particular, for the hypersurfaces being a cylinder over a corner or a cusp, the lists of simple "in the sense of Arnold" classes of singularities of function germs with respect to quasi border equivalence relations were obtained there, respectively.

In spite of a rather artificial nature of the definitions, quasi border singularities have very natural applications. Their discriminants reflect the behavior of critical points of a function (for example, of its global extremum) on domains with borders.

Arnold's classical boundary function singularity with $n$ parameters is related to a projection of a pair of $n$ dimensional Lagrangian submanifolds which have $(n-1)$-dimensional regular intersection [5]. The quasi border equivalence relation for generating families of functions keeps information only on one Lagrangian submanifold of the pair and on its intersection with the second component. So, it is a more natural model for applications involving Lagrangian submanifolds with boundary. Allowing singular boundaries, we arrive at a notion of a Lagrangian submanifold with a border. 
This is a pair $(L, \Gamma)$ consisting of a Lagrangian submanifold $L^{n} \subset M=T^{*} \mathbb{R}^{n}$ and of an $(n-1)$ dimensional isotropic variety $\Gamma \subset L$. Such an object arises in various singularity theory applications to differential equations and variational problems. Isotropic submanifolds play the role of the initial data set with some inequality constraints.

The quasi border bifurcation diagrams of function germ deformations consist of two components, $W_{0}$ and $W_{1}$. The $W_{0}$ is the ordinary discriminant which corresponds to all critical points of the deformation. The $W_{1}$ is a subset of $W_{0}$ which corresponds to the critical points on the border and satisfies extra equations defining the border. So the components have different dimensions. The caustic of a quasi border function deformation also consists of two strata or more. The first one is the ordinary caustic. The other stratum is the projection of the component $W_{1}$ of the bifurcation diagram to the base of the reduced deformation. The dimensions of these components of a caustic coincide. The geometry of bifurcation diagrams and caustics of simple quasi boundary and quasi corner singularities in $\mathbb{R}^{3}$ and $\mathbb{R}^{4}$ is described in [6].

The aim of the present paper is to consider a different type of a border. In particular, the border is cylinder over a swallowtail. The paper is organized as follows. In section 2 we recall the main definitions of the pseudo and quasi border equivalence relations from [4], and derive an expression of the quasi swallowtail tangent space. In section 3 we obtain the classifications of simple quasi swallowtail singularities. In section 4 the caustics and bifurcation diagrams of simple quasi swallowtail singularities are described. In section 5 we discuss the singularities of Lagrangian projections in presence of a swallowtail. Finally, in section 6 we finish the paper by a brief conclusion.

\section{PSEUDO AND QUASI BORDER EQUIVALENCE RELATIONS}

Consider a coordinate space $\mathbb{R}^{n}=\{w=(x, y)\}$ equipped with a cylindrical hypersurface $\Gamma$ given by the equation $B(x)=0$, where $x=\left(x_{1}, x_{2}, \ldots, x_{m}\right) \in \mathbb{R}^{m}$ and $y=\left(y_{1}, y_{2}, \ldots, y_{n-m}\right) \in \mathbb{R}^{n-m}$. We shall call $\Gamma$ a border.

The general statements below are valid for reasonably good borders. For rigorousness, we assume that the border is a stratified set, and the stratification satisfies the Whitney condition A.

We consider germs of $C^{\infty}$ functions $f:\left(\mathbb{R}^{n}, 0\right) \rightarrow \mathbb{R}$, in local coordinates $w$ as above. We denote by $\mathbf{C}_{w}$ the ring of all such germs at the origin and by $\mathcal{M}_{x, y}$ the maximal ideal in $\mathbf{C}_{x, y}$.

Definition 2.1 [4] Two functions $f_{0}, f_{1}: \mathbb{R}^{n} \rightarrow \mathbb{R}$ are called pseudo border equivalent if there exists a diffeomorphisim $\theta: \mathbb{R}^{n} \rightarrow \mathbb{R}^{n}$ such that $f_{1} \circ \theta=f_{0}$, and if a critical point $c$ of the function $f_{0}$ belongs to the border $\Gamma$ then $\theta(c)$ also belongs to $\Gamma$ and vice versa, if $c$ is a critical point of $f_{1}$ and belongs to $\Gamma$ then $\theta^{-1}(c)$ also belongs to $\Gamma$.

A similar definition can be introduced for germs of functions.

Remark 2.2 We assume in the definition 2.1 that if a critical point $c$ belongs to some stratum of $\Gamma$ then $\theta(c)$ belongs to the same stratum.

Clearly, the pseudo border equivalence is an equivalence relation: if $f_{1} \sim f_{2}$ and $f_{2} \sim f_{3}$ then $f_{1} \sim f_{3}$. However, this relation is not a group action as the set of admissible diffeomorphisms depends on a function. 
In the current paper, we consider the case when $\Gamma$ is a cylinder over a swallowtail. The swallowtail is realized as the subset $\Gamma=\Gamma_{s w l} \subset \mathbb{R}^{3}$ consisting of all points $\left\{\left(x_{1}, x_{2}, x_{3}\right)\right\}$ such that $z^{4}+x_{3} z^{2}+x_{2} z+x_{1}=0$ has multiple real roots. In this case the pseudo border equivalence will be also called pseudo swallowtail equivalence.

Recall that a vector field $v$ preserves a border $\Gamma=\{B(x)=0\}$ if the Lie derivative $L_{v} B$ belongs to the principal ideal generated by $B$. Such vector fields $v$ are tangent to $\Gamma$. The module $\mathbf{S}_{\Gamma}$ of all germs of $C^{\infty}$ vector fields preserving a germ $(\Gamma, 0) \subset\left(\mathbb{R}^{n}, 0\right)$ is the Lie algebra of the group of diffeomorphims of $\left(\mathbb{R}^{n}, 0\right)$ preserving $(\Gamma, 0)$. The module $\mathbf{S}_{\Gamma}$ is called the stationary algebra of $(\Gamma, 0)$. Due to classical Zakalyukin's algorithm [7] to write out basic vector fields tangent to the discriminant of an isolated function singularity, we have

$$
\begin{aligned}
\mathbf{S}_{\Gamma_{s w l}}= & \left\{\left[4 x_{1} h_{1}-\frac{x_{2} x_{3}}{2} h_{2}-\frac{3 x_{2}^{2}}{4} h_{3}\right] \frac{\partial}{\partial x_{1}}+\left[3 x_{2} h_{1}+\left(4 x_{1}-x_{3}^{2}\right) h_{2}-2 x_{2} x_{3} h_{3}\right] \frac{\partial}{\partial x_{2}}\right. \\
& \left.+\left[2 x_{3} h_{1}+3 x_{2} h_{2}+\left(4 x_{1}-x_{3}^{2}\right) h_{3}\right] \frac{\partial}{\partial x_{3}}+\sum_{i=1}^{n-3} k_{i} \frac{\partial}{\partial y_{i}}: h_{1}, h_{2}, h_{3}, k_{i} \in \mathbf{C}_{w}\right\} .
\end{aligned}
$$

Here $x=\left(x_{1}, x_{2}, x_{3}\right) \in \mathbb{R}^{3}$ and $y=\left(y_{1}, y_{2}, \ldots, y_{n-3}\right) \in \mathbb{R}^{n-3}$.

Definition $2.3[4]$ Let $J$ be an ideal in $\mathbf{C}_{w}$, then we define the $\operatorname{radical} \operatorname{Rad}(J)$ of the ideal $J$ as the set of all elements in $\mathbf{C}_{w}$, vanishing on the set of common zeros of germs from $J$ :

$$
\operatorname{Rad}(J)=I(V(J))
$$

where

$$
V(J)=\left\{w \in \mathbb{R}^{n}: h(w)=0 \text { for all } h \in J\right\},
$$

and

$$
I(V(J))=\left\{\varphi \in \mathbf{C}_{w}: \varphi(w)=0 \text { for all } w \in V(J)\right\}
$$

Remark 2.4 In general, the radical of an ideal behaves badly when the ideal depends on a parameter [4].

Suppose that all function germs in a smooth family $f_{t}$ are pseudo swallowtail equivalent to the function germ $f_{0}, f_{t} \circ \theta_{t}=f_{0}, t \in[0,1]$, with respect to a smooth family $\theta_{t}:\left(\mathbb{R}^{n}, 0\right) \rightarrow\left(\mathbb{R}^{n}, 0\right)$ of germs of diffeomorphisms such that $\theta_{0}=i d$ and $t \in[0,1]$. Then we have the homological equation:

$$
-\frac{\partial f_{t}}{\partial t}=\sum_{i=1}^{3} \frac{\partial f_{t}}{\partial x_{i}} \dot{X}_{i}(t)+\sum_{j=1}^{n-3} \frac{\partial f_{t}}{\partial y_{j}} \dot{Y}_{j}(t),
$$

where the vector field

$$
v_{t}=\sum_{i=1}^{3} \dot{X}_{i}(t) \frac{\partial}{\partial x_{i}}+\sum_{j=1}^{n-3} \dot{Y}_{j}(t) \frac{\partial}{\partial y_{j}}
$$

generates the phase flow $\theta_{t}$. 
Denote by $\operatorname{Rad}\left\{I_{t}\right\}$ the radical of the gradient ideal $I_{t}$ of the function $f_{t}$. Then by similar considerations to that in $[2,3,4]$, the components of $v_{t}$ satisfy the following:

$$
\begin{aligned}
& \dot{X}_{1}(t) \in\left\{4 x_{1} h_{1}-\frac{x_{2} x_{3}}{2} h_{2}-\frac{3 x_{2}^{2}}{4} h_{3}+\operatorname{Rad}\left\{I_{t}\right\}\right\}, \\
& \dot{X}_{2}(t) \in\left\{3 x_{2} h_{1}+\left(4 x_{1}-x_{3}^{2}\right) h_{2}-2 x_{2} x_{3} h_{3}+\operatorname{Rad}\left\{I_{t}\right\}\right\}, \\
& \dot{X}_{3}(t) \in\left\{2 x_{3} h_{1}+3 x_{2} h_{2}+\left(4 x_{1}-x_{3}^{2}\right) h_{3}+\operatorname{Rad}\left\{I_{t}\right\}\right\},
\end{aligned}
$$

and $\dot{Y}_{i}(t) \in \mathbf{C}_{w}$, where $h_{1}, h_{2}, h_{3} \in \mathbf{C}_{w}$.

Due to Remark 2.4 we modify the pseudo equivalence relation to have a better parameter dependence. Namely, we replace $\operatorname{Rad}\left\{I_{t}\right\}$ by the ideal $I_{t}$ itself in the equivalence definition. This provides us the notion of the quasi swallowtail equivalence.

Definition 2.5 Two functions $f_{0}, f_{1}: \mathbb{R}^{n} \rightarrow \mathbb{R}$ are called quasi swallowtail equivalent, if they are pseudo swallowtail equivalent and there is a family of functions $f_{t}$ which depends continuously on parameter $t \in[0,1]$ and a continuous piece-wise smooth family of diffeomorphisms $\theta_{t}: \mathbb{R}^{n} \rightarrow \mathbb{R}^{n}$ also depending on $t \in[0,1]$ such that: $f_{t} \circ \theta_{t}=f_{0}, \theta_{0}=i d$ and the components of the vector field $v_{t}$ generating $\theta_{t}$ on each segment of smoothness satisfy the following:

$$
\begin{aligned}
& \dot{X}_{1}(t) \in\left\{4 x_{1} h_{1}-\frac{x_{2} x_{3}}{2} h_{2}-\frac{3 x_{2}^{2}}{4} h_{3}+\left\{I_{t}\right\}\right\}, \\
& \dot{X}_{2}(t) \in\left\{3 x_{2} h_{1}+\left(4 x_{1}-x_{3}^{2}\right) h_{2}-2 x_{2} x_{3} h_{3}+\left\{I_{t}\right\}\right\}, \\
& \dot{X}_{3}(t) \in\left\{2 x_{3} h_{1}+3 x_{2} h_{2}+\left(4 x_{1}-x_{3}^{2}\right) h_{3}+\left\{I_{t}\right\}\right\},
\end{aligned}
$$

and $\dot{Y}_{i}(t) \in \mathbf{C}_{w}$, where $h_{1}, h_{2}, h_{3} \in \mathbf{C}_{w}$.

\section{Remarks 2.6}

1. Such a family $\theta_{t}$ of diffeomorphisms generated by the vector field $v_{t}$ as well as the vector field itself will be called admissible for the family $f_{t}$.

2. The tangent space $T Q S W L_{f}$ to the quasi swallowtail equivalence class of $f$ has the following description:

$$
\begin{aligned}
T Q S W L_{f} & =\left\{\frac{\partial f}{\partial x_{1}}\left(4 x_{1} h_{1}-\frac{x_{2} x_{3}}{2} h_{2}-\frac{3 x_{2}^{2}}{4} h_{3}+\sum_{i=1}^{3} \frac{\partial f}{\partial x_{i}} A_{i}\right)\right. \\
& +\frac{\partial f}{\partial x_{2}}\left(3 x_{2} h_{1}+\left(4 x_{1}-x_{3}^{2}\right) h_{2}-2 x_{2} x_{3} h_{3}+\sum_{i=1}^{3} \frac{\partial f}{\partial x_{i}} B_{i}\right) \\
& +\frac{\partial f}{\partial x_{3}}\left(2 x_{3} h_{1}+3 x_{2} h_{2}+\left(4 x_{1}-x_{3}^{2}\right) h_{3}+\sum_{i=1}^{3} \frac{\partial f}{\partial x_{i}} C_{i}\right) \\
& \left.+\sum_{i=1}^{n-3} \frac{\partial f}{\partial y_{i}} D_{i} \quad: \quad h_{1}, h_{2}, h_{3}, A_{i}, B_{i}, C_{i}, D_{i} \in \mathbf{C}_{w}\right\} .
\end{aligned}
$$


Due to the inclusion $I_{0}^{2} \subset T Q S W L_{f} \subset I_{0}$, where $I_{0}$ is the gradient ideal of $f$, we have

Proposition 2.7 A function germ $f$ has a finite codimension with respect to the quasi swallowtail equivalence if and only if $f$ has a finite codimension with respect to the right equivalence.

Definition 2.8 Two function germs are said to be stably quasi swallowtail equivalent if they become quasi swallowtail equivalent after the addition of non-degenerate quadratic forms in an appropriate number of extra cylindrical variables.

\subsection{Basic Techniques of the Classification and Prenormal Forms}

We will use Moser's homotopy method and the following technique which is essential for our normal form reduction in Section 3 to establish quasi swallowtail equivalence between function germs. It is similar to Lemma 8.1 in [3] and Lemma 2.10 in [4].

Let us fix a convenient Newton diagram $\Delta \subset \mathbb{Z}_{>0}^{n}$. The ideals $S_{\gamma}$ of function germs of the Newton order at least $\gamma, \gamma \geq 0$, equip the ring $\mathbf{C}_{w}$ with the Newton filtration: $S_{0}=\mathbf{C}_{w}, S_{\delta} \supset S_{\gamma}$ if $\delta<\gamma[8]$. We assume here that the scaling factor for the orders is chosen so that functions with the Newton diagram $\Delta$ have order $N$.

Let $f=f_{0}+f_{*}$ be a decomposition of a function germ $f$ into its principal part $f_{0}$ of the Newton degree $N$ and higher order terms $f_{*}$. We assume that $f_{0}$ has a finite codimension with respect to the right equivalence.

Lemma 2.9 Consider a monomial basis of the linear space $\mathbf{C}_{w} / T Q S W L_{f_{0}}$. Let $e_{1}(w), e_{2}(w)$, $\ldots, e_{s}(w)$ be all its elements of Newton degrees higher than $N$.

Suppose that for any $\varphi \in S_{\gamma} \backslash S_{>\gamma}, \gamma>N$ :

1. There is an admissible vector field $\dot{w}=\sum \dot{w}_{i} \frac{\partial}{\partial w_{i}}$ where $\dot{w}=\left(\dot{x}_{1}, \dot{x}_{2}, \dot{x}_{3}, \dot{y}_{1}, \dot{y}_{2}, \ldots, \dot{y}_{n-3}\right)$,

$$
\begin{gathered}
\dot{x}_{1}=4 x_{1} h_{1}-\frac{x_{2} x_{3}}{2} h_{2}-\frac{3 x_{2}^{2}}{4} h_{3}+\sum_{i=1}^{n} A_{1, i} \frac{\partial f_{0}}{\partial w_{i}}, \quad \dot{x}_{2}=3 x_{2} h_{1}+\left(4 x_{1}-x_{3}^{2}\right) h_{2}-2 x_{2} x_{3} h_{3}+\sum_{i=1}^{n} A_{2, i} \frac{\partial f_{0}}{\partial w_{i}}, \\
\dot{x}_{3}=2 x_{3} h_{1}+3 x_{2} h_{2}+\left(4 x_{1}-x_{3}^{2}\right) h_{3}+\sum_{i=1}^{n} A_{3, i} \frac{\partial f_{0}}{\partial w_{i}}, \quad \text { and } \quad \dot{y}_{1}, \ldots, \dot{y}_{n-3} \in \mathbf{C}_{w},
\end{gathered}
$$

with $h_{1}, h_{2}, h_{3}, A_{1, i}, A_{2, i}, A_{3, i} \in \mathbf{C}_{w}$, such that

$$
\varphi=\sum_{i=1}^{n} \frac{\partial f_{0}}{\partial w_{i}} \dot{w}_{i}+\widehat{\varphi}+\sum_{i=1}^{s} c_{i} e_{i}(w)
$$

where $\widehat{\varphi} \in S_{>\gamma}$ and $c_{i} \in \mathbb{R}$.

2. Moreover, for any $\delta, N<\delta<\gamma$, and any $\psi \in S_{\delta}$ the expression

$$
E(\psi, \varphi)=\sum_{i=1}^{3} \frac{\partial \psi}{\partial x_{i}}\left[\dot{x}_{i}+\sum_{j=1}^{n} A_{i, j} \frac{\partial \psi}{\partial w_{j}}\right]+2 \sum_{i=1}^{3} \frac{\partial f_{0}}{\partial x_{i}}\left[\sum_{j=1}^{n} A_{i, j} \frac{\partial \psi}{\partial w_{j}}\right]+\sum_{i=1}^{n-3} \frac{\partial \psi}{\partial y_{i}} \dot{y}_{i}
$$

belongs to $S_{\gamma}$. 
Then any germ $f=f_{0}+f_{*}$ is quasi swallowtail equivalent to a germ $f_{0}+\sum_{i=1}^{s} a_{i} e_{i}$, where $a_{i} \in \mathbb{R}$.

Remark 2.10 A version of the Lemma is also valid for functions with the Newton principal part $f_{0}$ of infinite right equivalence codimension, which is the same as having $\operatorname{dim}\left(\mathbf{C}_{w} / T Q S W L_{f_{0}}\right)$ $=\infty$. Namely, still assuming the Newton degree of $f_{0}$ being $N$, let $e_{1}(w), \ldots, e_{s}(w)$ be the degrees higher than $N$ part of a monomial basis of $\mathbf{C}_{w} /\left(T Q S W L_{f_{0}}+S_{M}\right)$ for some $M>N$. Assume the conditions of Lemma 2.9 hold for all $\gamma<M$. Then, any function with the Newton principal part $f_{0}$ is quasi swallowtail equivalent to

$$
f_{0}+\sum_{i=1}^{s} a_{i} e_{i}+\Psi, \quad \text { where } \quad \Psi \in S_{M} .
$$

\subsection{Quadratic Terms}

Let $f:\left(\mathbb{R}^{n}, 0\right) \rightarrow(\mathbb{R}, 0)$ be a function germ of the form

$$
f(x, y)=f_{2}(x, y)+f_{3}(x, y)
$$

where $f_{2}$ is a quadratic form in $x$ and $y$, and $f_{3} \in \mathcal{M}_{x, y}^{3}$.

Remark 2.11 A version of Lemma 2.12, Lemma 2.13, Lemma 2.14 and Lemma 2.15 in [4] are also true for functions when the cuspidal edge replaced by $\Gamma_{s w l}$. In fact, the components of the vector fields used there in their proofs are only from the gradient ideal of a family of functions. The phase flow of such vector fields leaves all critical points of the family fixed. Therefore, the family of diffeomorphisms generated by such vector fields is also admissible for quasi swallowtail equivalence. We state them below for $\Gamma_{s w l}$ with details of their proofs.

Lemma 2.12 Let $f:\left(\mathbb{R}^{3}, 0\right) \rightarrow(\mathbb{R}, 0)$ be a function germ at the origin in local coordinates $x_{1}, x_{2}$ and $x_{3}$ only. If $f_{2}$ is a non-degenerate quadratic form then $f$ is quasi swallowtail equivalent to $\pm x_{1}^{2} \pm x_{2}^{2} \pm x_{3}^{2}$.

Proof. Since $f_{2}$ is a non-degenerate quadratic form, vector fields with components from the gradient ideal of a function with a non-degenerate quadratic part are all vector fields vanishing at the origin. Therefore any family of diffeomorphisms preserving the origin is admissible, and the Lemma follows from the standard Morse Lemma.

Let $n \geq 3$ and set $f^{*}(y)=\left.f\right|_{x=0}$. Denote by $r^{*}$ the rank of the second differential $d_{0}^{2} f^{*}$ at the origin. Set $c=n-3-r^{*}$. Denote by $r$ the rank of the second differential $d_{0}^{2} f$ at the origin.

Lemma 2.13 (Stabilization) The function germ $f(x, y)$ is quasi swallowtail equivalent to a germ $\sum_{i=1}^{r^{*}} \pm y_{i}^{2}+g(x, \widetilde{y})$, where $\widetilde{y} \in \mathbb{R}^{c}$ and $g^{*} \in \mathcal{M}_{\widetilde{y}}^{3}$. For quasi swallowtail equivalent germs $f$, the respective reduced germs $g$ are quasi swallowtail equivalent.

Proof. Up to a linear transformation in $y$, we have

$$
f=\sum_{i=1}^{r^{*}} \pm y_{i}^{2}+\sum_{i=1}^{n-3} \sum_{j=1}^{3} a_{i, j} y_{i} x_{j}+Q_{2}(x)+f_{3}(x, y),
$$


with $f_{3} \in \mathcal{M}_{x, y}^{3}$ and $Q_{2}$ a quadratic form in $x$ only.

Let $\hat{y}=\left(y_{1}, y_{2}, \ldots, y_{r^{*}}\right)$ and $\widetilde{y}=\left(y_{r^{*}+1}, \ldots, y_{n-3}\right) \in \mathbb{R}^{n-3-r^{*}}$. Then, (1) can be written as

$$
f_{1}=\sum_{i=1}^{r^{*}} \pm y_{i}^{2}+\varphi(x, \hat{y}, \widetilde{y})+\widetilde{f}(x, \widetilde{y})
$$

where

$$
\varphi=\sum_{i=1}^{r^{*}} \sum_{j=1}^{3} a_{i, j} y_{i} x_{j}+\sum_{l=1}^{r^{*}} y_{l} \widetilde{\varphi}_{l}(x, y) \quad \text { with } \quad \widetilde{\varphi}_{l} \in \mathcal{M}_{x, y}^{2}
$$

and

$$
\widetilde{f}=Q_{2}(x)+\sum_{i=r^{*}+1}^{n-3} \sum_{j=1}^{3} a_{i, j} y_{i} x_{j}+\widetilde{f}_{3}(x, \widetilde{y}) \quad \text { with } \quad \widetilde{f}_{3} \in \mathcal{M}_{x, \widetilde{y}}^{3}
$$

We now aim to find a family

$$
\theta_{t}:(x, y) \mapsto\left(x, \hat{Y}_{t}(x, \hat{y}), \widetilde{y}\right)
$$

of diffeomorphisms which eliminates $\varphi$.

Take a family $f_{t}=\sum_{i=1}^{r^{*}} \pm y_{i}^{2}+t \varphi(x, \hat{y}, \widetilde{y})+\widetilde{f}_{t}(x, \widetilde{y})$ which joins $f_{1}$ and $f_{0}=\sum_{i=1}^{r^{*}} \pm y_{i}^{2}+\widetilde{f}_{0}(x, \widetilde{y})$ with $t \in[0,1]$ and $\widetilde{f}=\widetilde{f}_{1}$. Here, $\widetilde{f}_{t}$ and $\widetilde{f}_{0}$ are unknown. So, we want to solve the homological equation for $\dot{y}$ and simultaneously for $\widetilde{f}_{t}$.

The homological equation takes the form

$$
-\frac{\partial f_{t}}{\partial t}=\sum_{i=1}^{3} \frac{\partial f_{t}}{\partial x_{i}} \dot{x}_{i}+\sum_{i=1}^{r^{*}} \frac{\partial f_{t}}{\partial y_{i}} \dot{y}_{i}+\sum_{j=r^{*}+1}^{n-3} \frac{\partial f_{t}}{\partial y_{j}} \dot{y}_{j} .
$$

Note that $\dot{x}_{1}=\dot{x}_{2}=\dot{x}_{3}=\dot{y}_{j}=0, j=r^{*}+1, \ldots, n-3$, as $x$ and $\widetilde{y}$ do not change with $t$.

Thus, (2) can be written as

$$
-\left(\varphi+\frac{\partial \tilde{f}_{t}}{\partial t}\right)=\sum_{i=1}^{r^{*}}\left( \pm 2 y_{i}+t \frac{\partial \varphi}{\partial y_{i}}\right) \dot{y}_{i}
$$

Set $z_{i}= \pm 2 y_{i}+t \frac{\partial \varphi}{\partial y_{i}}, i=1, \ldots, r^{*}$, which are known functions. Note that the matrix $\left(\frac{\partial z_{i}}{\partial \hat{y}_{j}}\right)$ has the maximal rank at the origin for any value of $t$. Hence we can take $z=\left(z_{1}, z_{2}, \ldots, z_{r^{*}}\right)$ as new coordinates instead of $\hat{y}$. Thus, (3) takes the form

$$
-\left(\varphi+\frac{\partial \widetilde{f}_{t}}{\partial t}\right)=\sum_{i=1}^{r^{*}} z_{i} \dot{y}_{i}
$$


Using the Hadamard Lemma, we write this as

$$
\sum_{i=1}^{r^{*}} z_{i} \psi_{i}(x, z, \widetilde{y}, t)+\phi(x, \widetilde{y}, t)+\frac{\partial \tilde{f}_{t}}{\partial t}=\sum_{i=1}^{r^{*}}-z_{i} \dot{y}_{i} .
$$

By taking $\psi_{i}=-\dot{y}_{i}$ and $\frac{\partial \widetilde{f}_{t}}{\partial t}=-\phi$, we show that the homological equation is solvable.

The last step is to find $\widetilde{f}_{0}$. This can be done using the relation

$$
-\int_{0}^{1} \phi d t=\int_{0}^{1} \frac{\partial \widetilde{f}_{t}}{\partial t} d t=\widetilde{f}_{1}-\widetilde{f}_{0}
$$

Note that the vector field $\dot{v}=\sum_{i=1}^{r^{*}} \dot{y}_{i} \frac{\partial}{\partial y_{i}}$ is defined in some neighborhood of the segment $[0,1]$ of the $t$-axis in the space $\mathbb{R}^{n} \times \mathbb{R}_{t}$, which is due to the $z_{i}$ vanishing on this segment.

Hence all the $f_{t}$ are quasi swallowtail equivalent. In particular, the function germ $f_{1}$ is quasi swallowtail equivalent to $f_{0}$.

The second claim of the Lemma can be deduced directly as the family

$$
\theta_{t}:(x, y) \mapsto\left(x, \hat{Y}_{t}(x, \hat{y}), \widetilde{y}\right)
$$

preserves the projection $(x, \hat{y}, \widetilde{y}) \mapsto(x, \widetilde{y})$.

Lemma 2.14 Let $f:\left(\mathbb{R}^{n}, 0\right) \rightarrow \mathbb{R}$ be a function germ with an isolated critical point at the origin, and $I_{0}$ its gradient ideal. Then $f$ is quasi swallowtail equivalent for each $t \in[0,1]$ to the function germ $g_{t}(w)=f(w)+$ th $(w)$ with $h(w) \in I_{0}^{2}$, provided that the rank $r$ of the second differential $d_{0}^{2} g_{t}$ of $g_{t}$ at the origin is constant.

Proof. At first we claim that if the rank of $d_{0}^{2} g_{t}$ is constant then for different $t$ the gradient ideals $I_{t}$ of $g_{t}$ coincide. Since the claim does not depend on the choice of local coordinates, we may assume that the quadratic part of $f$ at the origin has diagonal form $\sum_{i=1}^{r} \varepsilon_{i} w_{i}^{2}$, where $\varepsilon_{i}= \pm 1$ for $i=1, \ldots, r$. We also set $\varepsilon_{i}=0$ for $i>r$.

The quadratic part of $g_{t}$ at the origin is

$$
\sum_{i, j=1}^{r}\left(\varepsilon_{i} \delta_{i j} w_{i}^{2}+4 t h_{i j}(0) \varepsilon_{i} \varepsilon_{j} w_{i} w_{j}\right)=\sum_{i, j=1}^{r} D_{i j} w_{i} w_{j}
$$

where the $h_{i j}, i, j=1, \ldots, n$ are the coefficients of the decomposition

$$
h(w)=\sum_{i, j=1}^{n} h_{i j}(w) \frac{\partial f}{\partial w_{i}} \frac{\partial f}{\partial w_{j}}
$$

of the function $h, \delta_{i j}$ is the Kronecker symbol, and $D_{i j}=\varepsilon_{i} \delta_{i j}+4 t \varepsilon_{i} \varepsilon_{j} h_{i j}(0)$. We will assume here that $h_{i j}=h_{j i}$. 
The $r \times r$ matrix with entries $D_{i j}$ is invertible for any $t$ since the rank of $d_{0}^{2} g_{t}$ is $r$. Reversing signs of some of its rows, we see that the $n \times n$ matrix with the entries $\hat{D}_{i j}=\delta_{i j}+4 t \varepsilon_{j} h_{i j}(0)$, for $i, j=1, \ldots, r$ and $\hat{D}_{i j}=\delta_{i j}$ otherwise, is also invertible.

The differentiation

$$
\frac{\partial g_{t}}{\partial w_{i}}=\frac{\partial f}{\partial w_{i}}+t \sum_{k, j=1}^{n}\left(2 h_{k j} \frac{\partial^{2} f}{\partial w_{k} \partial w_{i}}+\frac{\partial h_{k j}}{\partial w_{i}} \frac{\partial f}{\partial w_{k}}\right) \frac{\partial f}{\partial w_{j}}
$$

implies that $I_{t} \subset I_{0}$. This derivative can also be written as

$$
\frac{\partial g_{t}}{\partial w_{i}}=\sum_{j=1}^{n}\left(\delta_{i j}+4 t \varepsilon_{i} h_{i j}(0)+R_{j i}\right) \frac{\partial f}{\partial w_{j}}=\sum_{j=1}^{n}\left(\hat{D}_{j i}+R_{j i}\right) \frac{\partial f}{\partial w_{j}},
$$

where the functions $R_{i j}$ vanish at $w=0$. So in some neighborhood of the interval $[0,1]$ of the $t$-axis the matrix $\left(\hat{D}_{j i}+R_{j i}\right)$ is invertible. This implies that $I_{0} \subset I_{t}$. Hence, $I_{t}=I_{0}$.

Now the homological equation $-\frac{\partial g_{t}}{\partial t}=\sum_{i=1}^{n} \frac{\partial g_{t}}{\partial w_{i}} V_{i}$ can be solved for the unknown functions $V_{i}$ which belong to the gradient ideal $I_{t}$ for any $t$, since the left hand side belongs to the square of this ideal. The phase flow of the vector field $\sum V_{i} \frac{\partial}{\partial w_{i}}$ leaves all critical points of $g_{t}$ fixed. Hence all the germs $g_{t}$ are quasi swallowtail equivalent.

Lemmas 2.13 and 2.14 imply the following improved stabilization splitting.

Lemma 2.15 There is a non-negative integer $s \leq r-r^{*}$ such that the function germ $f(x, y)$ is quasi swallowtail equivalent to $\sum_{i=1}^{r^{*}+s} \pm y_{i}^{2}+\widetilde{f}(x, \widetilde{y})$, where $\widetilde{y} \in \mathbb{R}^{c-s}$ and $\widetilde{f}$ is a sum of a function germ from $\mathcal{M}_{x, \widetilde{y}}^{3}$ and a quadratic form in $x$ only. For quasi swallowtail equivalent germs $f$, the corresponding reduced germs $\tilde{f}$ are quasi swallowtail equivalent.

Proof. Due to Lemma 2.13, we can assume that the quadratic part of the function is $f_{2}=\sum_{i=1}^{r^{*}} \pm y_{i}^{2}+x_{1} \sum_{i=r^{*}+1}^{n-3} \alpha_{1, i} y_{i}+x_{2} \sum_{i=r^{*}+1}^{n-3} \alpha_{2, i} y_{i}+x_{3} \sum_{i=r^{*}+1}^{n-3} \alpha_{3, i} y_{i}+g_{2}(x)$ with constant coefficients $\alpha_{j, i}$ and the quadratic form $g_{2}$ in $x$ only. Suppose that some of these coefficients, for example $\alpha_{1, r^{*}+1}$, is non-zero. Then, summing up the function $f$ with $\delta\left(\frac{\partial f}{\partial x_{1}}\right)^{2}$ for sufficiently small $\delta$ gives a new function $g$ which (according to Lemma 2.14) is quasi swallowtail equivalent to $f$ and contains the term $y_{r^{*}+1}^{2}$ with a non-zero coefficient. Therefore the rank of the restriction of $g$ to the $x=0$ subspace is larger than $r^{*}$. Repeating the procedure several times, if needed, we get a function germ with a larger value of $r^{*}$ and without the $x_{j} y_{>r^{*}}$ terms. This is exactly the form required.

\section{CLASSIFICATION OF SIMPLE FUNCTIONS}

Following [8], we call a function germ simple if its sufficiently small neighbourhood in the space of all function germs contains only a finite number of quasi equivalence classes.

The quasi swallowtail classification of critical points outside the border $\Gamma_{s w l}$ coincides with the standard right equivalence. Hence the standard classes $A_{k}, D_{k}, E_{6}, E_{7}$ and $E_{8}$ form the list of simple classes in this case. Also by definition, non-critical points are all equivalent wherever they are. When the critical point of a function germ lies on $\Gamma_{s w l}$ then we distinguish the following cases: 
- If the base point is at a regular point of $\Gamma_{s w l}$, then the quasi swallowtail equivalence coincides with the quasi boundary equivalence. Hence, the list of simple quasi swallowtail classes in this case is the same as that of simple quasi boundary classes [2].

- If the base point belongs to the self-interesting stratum of $\Gamma_{s w l}$, then the quasi swallowtail equivalence coincides with the quasi corner equivalence. Hence, the list of simple quasi swallowtail classes in this case is the same as that of simple quasi corner classes[3].

- If the base point belongs to the cusp stratum of $\Gamma_{s w l}$, then the list of simple quasi swallowtail classes is the same as that of simple quasi corner classes [4].

- The remaining case of a function germ having a swallowtail point is described by the following theorem.

Theorem 3.1 Let a function germ $f:\left(\mathbb{R}^{n}, 0\right) \rightarrow(\mathbb{R}, 0)$, be simple with respect to the quasi swallowtail equivalence. Then, either its quadratic part $f_{2}$ is non-degenerate and hence $f$ is quasi swallowtail equivalent to $\mathcal{Q}_{2}: \pm x_{1}^{2} \pm x_{2}^{2} \pm x_{3}^{2}+\sum_{i=1}^{n-3} \pm y_{i}^{2}$ or $f_{2}$ has corank 1 in which case $f$ is stably quasi swallowtail equivalent to one of the following simple classes:

\begin{tabular}{|c|c|c|c|}
\hline Notation & Normal form & Restrictions & Codimension \\
\hline $\mathcal{Q}_{k}$ & $\pm x_{2}^{2} \pm x_{3}^{2} \pm x_{1}^{k}$ & $k \geq 3$ & $k+2$ \\
\hline $\mathcal{T}_{k}$ & $\pm x_{1}^{2} \pm x_{3}^{2} \pm x_{2}^{k}$ & $k \geq 3$ & $k+3$ \\
\hline $\mathcal{D}_{3}$ & $\pm x_{1}^{2} \pm x_{2}^{2}+x_{3}^{3}$ & & 7 \\
\hline $\mathcal{N}_{2,2,2, k}$ & $\pm\left(x_{1}+y_{1}^{2}\right)^{2} \pm\left(x_{2}+y_{1}^{2}\right)^{2} \pm\left(x_{3}+y_{1}^{2}\right)^{2} \pm y_{1}^{k}$ & $k \geq 3$ & $k+5$ \\
\hline $\mathcal{N}_{3,2,2,4}$ & $\pm\left(x_{1}+y_{1}^{3}\right)^{2} \pm\left(x_{2}+y_{1}^{2}\right)^{2} \pm\left(x_{3}+y_{1}^{2}\right)^{2} \pm y_{1}^{4}$ & & 10 \\
\hline $\mathcal{N}_{3,3,2,4}$ & $\pm\left(x_{1}+y_{1}^{3}\right)^{2} \pm\left(x_{2}+y_{1}^{3}\right)^{2} \pm\left(x_{3}+y_{1}^{2}\right)^{2} \pm y_{1}^{4}$ & & 11 \\
\hline $\mathcal{N}_{3,3,3,4}$ & $\pm\left(x_{1}+y_{1}^{3}\right)^{2} \pm\left(x_{2}+y_{1}^{3}\right)^{2} \pm\left(x_{3}+y_{1}^{3}\right)^{2} \pm y_{1}^{4}$ & & 12 \\
\hline
\end{tabular}

\section{Remarks 3.2}

1. Any germ $f$ with the quadratic part of corank greater than 1 is non-simple.

2. Any germ of corank 1 is either simple (and hence quasi swallowtail equivalent to one of the germs in the above theorem) or belongs to a subset of infinite codimension in the space of all germs.

3. The fencing classes are stabilizations of the following:

\begin{tabular}{|c|c|c|c|}
\hline Notation & Class & Restrictions & Codimension \\
\hline$\widetilde{\mathcal{Q}}$ & $\pm\left(x_{1}+x_{2}+x_{3}\right)^{2} \pm x_{1}^{3}+\alpha x_{3}^{3}$ & $\alpha \in \mathbb{R} \backslash\{0\}$ & 8 \\
\hline $\mathcal{D}_{4}$ & $\pm x_{2}^{2} \pm\left(x_{1}+\delta x_{3}^{2}\right)^{2} \pm x_{3}^{4}$, & $\delta \in \mathbb{R}$ & 8 \\
\hline $\mathcal{N}_{3,2,2,5}$ & $\pm\left(x_{1}+y_{1}^{3}\right)^{2} \pm\left(x_{2}+y_{1}^{2}\right)^{2}+ \pm\left(x_{2}+y_{1}^{2}\right)^{2}+\beta y_{1}^{5}$ & $\beta \in \mathbb{R} \backslash\{0\}$ & 11 \\
\hline $\mathcal{N}_{3,3,2,5}$ & $\pm\left(x_{1}+y_{1}^{3}\right)^{2} \pm\left(x_{2}+y_{1}^{3}\right)^{2} \pm\left(x_{2}+y_{1}^{2}\right)^{2} \gamma y_{1}^{5}$ & $\gamma \in \mathbb{R} \backslash\{0\}$ & 12 \\
\hline
\end{tabular}


4. The graph of adjacencies in low codimension is as follows:

$$
\begin{aligned}
& \mathcal{Q}_{2} \leftarrow \mathcal{Q}_{3} \leftarrow \mathcal{Q}_{4} \leftarrow \mathcal{Q}_{5} \leftarrow \mathcal{Q}_{6} \leftarrow \ldots
\end{aligned}
$$

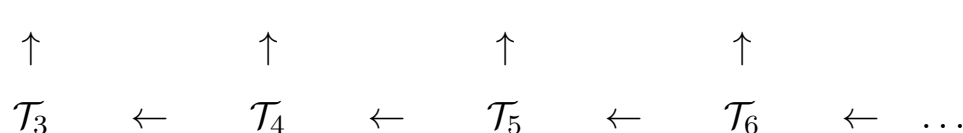

$$
\begin{aligned}
& \mathcal{D}_{3} \leftarrow \mathcal{D}_{4} \\
& \uparrow \\
& \mathcal{N}_{2,2,2,3} \leftarrow \mathcal{N}_{2,2,2,4} \leftarrow \mathcal{N}_{2,2,2,5} \leftarrow \mathcal{N}_{2,2,2,6} \leftarrow \ldots \\
& \uparrow \quad \uparrow \\
& \mathcal{N}_{3,2,2,4} \leftarrow \mathcal{N}_{3,2,2,5} \\
& \uparrow \quad \uparrow \\
& \mathcal{N}_{3,3,2,4} \leftarrow \mathcal{N}_{3,3,2,5} \\
& \uparrow \\
& \mathcal{N}_{3,3,3,4}
\end{aligned}
$$

Also, $\mathcal{D}_{3} \leftarrow \widetilde{\mathcal{Q}}$.

To prove Theorem 3.1, we need the following auxiliary results.

Lemma 3.3 Let $\kappa=n-r$ be the corank of the second differential $d_{0}^{2} f$ at the origin.

1. If $\kappa=0$, then $f$ is quasi swallowtail equivalent to $\sum_{i=1}^{n-3} \pm y_{i}^{2}+f_{2}(x)+f_{3}(x)$, where $f_{2}$ is a non-degenerate quadratic form and $f_{3} \in \mathcal{M}_{x}^{3}$.

2. If $\kappa=1$, then $f$ is quasi swallowtail equivalent to either $\sum_{i=1}^{n-3} \pm y_{i}^{2}+\widetilde{f}(x)$ with $\operatorname{rank}\left(d_{0}^{2} \widetilde{f}\right)=2$ or to $\sum_{i=2}^{n-3} \pm y_{i}^{2} \pm x_{1}^{2} \pm x_{2}^{2} \pm x_{3}^{2}+f_{3}\left(x, y_{1}\right)$, where $f_{3}\left(x, y_{1}\right) \in \mathcal{M}_{x, y_{1}}^{3}$.

3. If $\kappa \geq 2$, then $f$ is non-simple.

Proof. Lemmas 2.12 and 2.15 provide the first two parts of Lemma 3.3.

For part 3 , suppose that $\kappa=2$. Then Lemma 2.15 yields that any function germ $f(x, y)$ reduced to one of the following forms:

0. $\quad F_{0}=\sum_{i=1}^{n-3} \pm y_{i}^{2}+f_{2}(x)+f_{3}(x)$ where $f_{3} \in \mathcal{M}_{x}^{3}$, and $f_{2}$ is a quadratic form in $x$ of rank one, or

1. $\quad F_{1}=\sum_{i=2}^{n-3} \pm y_{i}^{2}+f_{2}(x)+f_{3}\left(x, \widetilde{y}_{1}\right)$ where $f_{3} \in \mathcal{M}_{x, \widetilde{y}_{1}}^{3}, \widetilde{y}_{1} \in \mathbb{R}$ and $f_{2}$ is a quadratic form in $x$ of rank two, or

2. $\quad F_{2}=\sum_{i=3}^{n-3} \pm y_{i}^{2}+f_{2}(x)+f_{3}(x, \tilde{y})$ where $f_{3} \in \mathcal{M}_{x, \widetilde{y}}^{3}, \widetilde{y}=\left(\widetilde{y}_{1}, \widetilde{y}_{2}\right)$ and $f_{2}$ is a non-degenerate 
quadratic form in $x$.

Consider the germ $F_{0}$. Then we may write $F_{0}=\sum_{i=1}^{n-3} \pm y_{i}^{2} \pm\left(a x_{1}+b x_{2}+c x_{3}\right)^{2}+f_{3}$ where $f_{3} \in \mathcal{M}_{x}^{3}$, and $a, b, c \in \mathbb{R}(a, b$, and $c$ are not all zeros simultaneously). Suppose $a \neq 0$ and set $\widetilde{x}_{1}=a x_{1}+b x_{2}+c x_{3}$. Hence we can take $\widetilde{x}_{1}$ as a new coordinate instead of $x_{1}$. Thus, up to linear transformation in $\widetilde{x}_{1}, x_{2}$ and $x_{3}$, we have

$$
F_{0} \sim \widetilde{F}=\sum_{i=1}^{n-3} \pm y_{i}^{2} \pm \widetilde{x}_{1}^{2}+\widetilde{f}_{3}, \quad \widetilde{f}_{3} \in M_{\widetilde{x}_{1}, x_{2}, x_{3}}^{3} .
$$

The tangent space to the quasi swallowtail orbit at the germ $\widetilde{F}$ in the new coordinates is

$$
\begin{aligned}
T Q S W L_{\widetilde{F}} & =\left\{\frac{\partial \widetilde{F}}{\partial \widetilde{x}_{1}}\left[\frac{4}{a}\left(\widetilde{x}_{1}-b x_{2}-c x_{3}\right) h_{1}-\frac{x_{2} x_{3}}{2} h_{2}-\frac{3 x_{2}^{2}}{4} h_{3}+\frac{\partial \widetilde{F}}{\partial \widetilde{x}_{1}} A_{1}+\sum_{i=2}^{3} \frac{\partial \widetilde{F}}{\partial x_{i}} A_{i}\right]\right. \\
& +\frac{\partial \widetilde{F}}{\partial x_{2}}\left[3 x_{2} h_{1}+\left(\frac{4}{a}\left(\widetilde{x}_{1}-b x_{2}-c x_{3}\right)-x_{3}^{2}\right) h_{2}-2 x_{2} x_{3} h_{3}+\frac{\partial \widetilde{F}}{\partial \widetilde{x}_{1}} B_{1}+\sum_{i=2}^{3} \frac{\partial \widetilde{F}}{\partial x_{i}} B_{i}\right] \\
& \left.+\frac{\partial \widetilde{F}}{\partial x_{3}}\left[2 x_{3} h_{1}+3 x_{2} h_{2}+\left(\frac{4}{a}\left(\widetilde{x}_{1}-b x_{2}-c x_{3}\right)-x_{3}^{2}\right) h_{3}+\frac{\partial \widetilde{F}}{\partial \widetilde{x}_{1}} C_{1}+\sum_{i=2}^{3} \frac{\partial \widetilde{F}}{\partial x_{i}} C_{i}\right]\right\} .
\end{aligned}
$$

Now, consider the principal part $\widetilde{F}_{0}= \pm \widetilde{x}_{1}$ of $\widetilde{F}$. The tangent space to the quasi swallowtail orbit at $\widetilde{F}_{0}$ is

$$
T Q S W L_{\widetilde{F}_{0}}=\left\{ \pm 2 \widetilde{x}_{1}\left[\frac{4}{a}\left(\widetilde{x}_{1}-b x_{2}-c x_{3}\right) h_{1}-\frac{x_{2} x_{3}}{2} h_{2}-\frac{3 x_{2}^{2}}{4} h_{3}+\frac{\partial \widetilde{F}}{\partial \widetilde{x}_{1}} A_{1}+\sum_{i=2}^{3} \frac{\partial \widetilde{F}}{\partial x_{i}} A_{i}\right]\right\} .
$$

Thus, we get $\bmod T Q S W L_{\widetilde{F}_{0}}: \widetilde{x}_{1}^{2} \equiv 0, \widetilde{x}_{1}\left(-b x_{2}-c x_{3}\right) \equiv 0, \widetilde{x}_{1} x_{2} x_{3} \equiv 0$, and $\widetilde{x}_{1} x_{2}^{2} \equiv 0$. Therefore, we have $\mathbf{C}_{\widetilde{x}_{1}, x_{2}, x_{3}} / T Q S W L_{\widetilde{F}_{0}} \simeq\left\{\alpha \widetilde{x}_{1}+\beta \widetilde{x}_{1} x_{2}+\varphi\left(x_{2}, x_{3}\right): \alpha, \beta \in \mathbb{R}, \varphi \in \mathbf{C}_{x_{2}, x_{3}}\right\}$. According to Remark 2.10, the germ $\widetilde{F}$ is quasi swallowtail equivalent to

$$
\pm \widetilde{x}_{1}^{2}+g\left(x_{2}, x_{3}\right) \quad \text { with } \quad g \in \mathcal{M}_{x_{2}, x_{3}}^{3} .
$$

Let $H=\widetilde{a} \widetilde{x}_{1}^{2}+a_{30} x_{2}^{3}+a_{21} x_{2}^{2} x_{3}+a_{12} x_{2} x_{3}^{2}+a_{03} x_{3}^{3}$ be the lowest quasi homogenous part of $(*)$ with respect to the weights: $w_{\widetilde{x}_{1}}=3, w_{x_{2}}=w_{x_{3}}=2$. The tangent space to the quasi swallowtail orbit at $H$ is

$$
\begin{aligned}
T Q S W L_{H} & =\left\{\frac{\partial H}{\partial \widetilde{x}_{1}}\left[\frac{4}{a}\left(\widetilde{x}_{1}-b x_{2}-c x_{3}\right) h_{1}-\frac{x_{2} x_{3}}{2} h_{2}-\frac{3 x_{2}^{2}}{4} h_{3}+\frac{\partial H}{\partial \widetilde{x}_{1}} A_{1}+\sum_{i=2}^{3} \frac{\partial H}{\partial x_{i}} A_{i}\right]\right. \\
& +\frac{\partial H}{\partial x_{2}}\left[3 x_{2} h_{1}+\left(\frac{4}{a}\left(\widetilde{x}_{1}-b x_{2}-c x_{3}\right)-x_{3}^{2}\right) h_{2}-2 x_{2} x_{3} h_{3}+\frac{\partial H}{\partial \widetilde{x}_{1}} B_{1}+\sum_{i=2}^{3} \frac{\partial \widetilde{F}}{\partial x_{i}} B_{i}\right] \\
& \left.+\frac{\partial H}{\partial x_{3}}\left[2 x_{3} h_{1}+3 x_{2} h_{2}+\left(\frac{4}{a}\left(\widetilde{x}_{1}-b x_{2}-c x_{3}\right)-x_{3}^{2}\right) h_{3}+\frac{\partial H}{\partial \widetilde{x}_{1}} C_{1}+\sum_{i=2}^{3} \frac{\partial H}{\partial x_{i}} C_{i}\right]\right\} .
\end{aligned}
$$


The terms of $H$ in $T Q S W L_{f_{2}}$ are from

$$
\begin{gathered}
{\left[\left(\frac{4}{a}\left(\widetilde{x}_{1}-b x_{2}-c x_{3}\right)\right) \frac{\partial H}{\partial \widetilde{x}_{1}}+3 x_{2} \frac{\partial H}{\partial x_{2}}+2 x_{3} \frac{\partial H}{\partial x_{3}}\right] h_{1}} \\
{\left[-\frac{x_{2} x_{3}}{2} \frac{\partial H}{\partial \widetilde{x}_{1}}+\left(\frac{4}{a}\left(\widetilde{x}_{1}-b x_{2}-c x_{3}\right)-x_{3}^{2}\right) \frac{\partial H}{\partial x_{2}}+3 x_{2} \frac{\partial H}{\partial x_{3}}\right] h_{2},} \\
{\left[-\frac{3 x_{2}^{2}}{4} \frac{\partial H}{\partial \widetilde{x}_{1}}-2 x_{2} x_{3} \frac{\partial H}{\partial x_{2}}+\left(\frac{4}{a}\left(\widetilde{x}_{1}-b x_{2}-c x_{3}\right)-x_{3}^{2}\right) \frac{\partial H}{\partial x_{3}}\right] h_{3},}
\end{gathered}
$$

and

$$
\left(\frac{\partial H}{\partial \widetilde{x}_{1}}\right)^{2} A_{1}
$$

These terms form a subspace of dimension at most 4 . The dimension of the space $L=\left\{\left(\widetilde{a}, a_{30}, a_{21}, a_{12}, a_{03}\right)\right\}$ of coefficients of $H$ is 5 which is greater than the subspace dimension. This means that the germ $F_{0}$ is non-simple.

By similar arguments we can show that $F_{0}$ is non-simple when $a=0$ and $b \neq 0$ or $a=b=0$ and $c \neq 0$.

In the next case we have $F_{1}=\sum_{i=2}^{n-3} \pm y_{i}^{2} \pm\left(a_{1} x_{1}+b_{1} x_{2}+c_{1} x_{3}\right)^{2}+\left(a_{2} x_{1}+b_{2} x_{2}+c_{2} x_{3}\right)^{2}+f_{3}$, where $f_{3} \in \mathcal{M}_{x, \widetilde{y}_{1}}$, and $a_{i}, b_{i}, c_{i} \in \mathbb{R}\left(a_{1}, b_{1}, c_{1}\right.$ or $a_{2}, b_{2}, c_{2}$ are not all zeros simultaneously). Note that $F_{1}$ deforms to

$$
\widetilde{F}_{1}=\sum_{i=2}^{n-3} \pm y_{i}^{2} \pm\left(a_{1} x_{1}+b_{1} x_{2}+c_{1} x_{3}\right)^{2}+\left(a_{2} x_{1}+b_{2} x_{2}+c_{2} x_{3}+\delta \widetilde{y}_{1}\right)^{2}+f_{3}, \quad \delta \neq 0 .
$$

According to Lemma $2.15, \widetilde{F}_{1}$ is quasi swallowtail equivalent to $\sum_{i=1}^{n-3} \pm y_{i}^{2}+ \pm\left(a x_{1}+b x_{2}+c x_{3}\right)^{2}+\widetilde{f}$ with $\tilde{f} \in \mathcal{M}_{x}^{3}$, which we have already shown to be non-simple. Similar argument yields that $F_{2}$ is adjacent to $F_{1}$ and the result follows.

Lemma 3.4 Let $f:\left(\mathbb{R}^{3}, 0\right) \mapsto(\mathbb{R}, 0)$ be a function germ in local coordinates $x_{1}, x_{2}$ and $x_{3}$, and with a critical point at the origin. If the quadratic form $f_{2}$ of $f$ has rank 2 then $f$ is quasi swallowtail equivalent to either $\pm x_{2}^{2} \pm x_{3}^{2}+\varphi_{1}\left(x_{1}\right)$ where $\varphi_{1} \in \mathcal{M}_{x_{1}}^{3}$ or $\pm x_{1}^{2} \pm x_{3}^{2}+\varphi_{2}\left(x_{2}\right)$ where $\varphi_{2} \in \mathcal{M}_{x_{2}}^{3}$ or $\pm x_{1}^{2} \pm x_{2}^{2}+x_{1} \varphi_{3}\left(x_{3}\right)+\varphi_{4}\left(x_{3}\right)$ where $\varphi_{3} \in \mathcal{M}_{x_{3}}^{2}$ and $\varphi_{4} \in \mathcal{M}_{x_{3}}^{3}$.

Proof. The hierarchy of lines in $T_{0} \mathbb{R}^{3}$ with respect to diffeomorphisms preserving the swallowtail is

$$
x_{1} \text {-axis } \leftarrow x_{2} \text {-axis } \leftarrow x_{3} \text {-axis. }
$$

Considering them as kernals of quadratic forms, we get the hierarchy of corank 2 forms

$$
\pm x_{2}^{2} \pm x_{3}^{2} \leftarrow \pm x_{1}^{2} \pm x_{3}^{2} \leftarrow \pm x_{1}^{2} \pm x_{2}^{2} .
$$

Consider the germ $G= \pm x_{2}^{2} \pm x_{3}^{2}+g_{3}(x)$, where $g_{3} \in \mathcal{M}_{x}^{3}$. Let $G_{0}= \pm x_{2}^{2} \pm x_{3}^{2}$. The quasi swallowtail tangent space at $G_{0}$ is

$$
\begin{aligned}
\text { TQSWL } L_{0}= & \left\{ \pm 2 x_{2}\left[3 x_{2} h_{1}+\left(4 x_{1}-x_{3}^{2}\right) h_{2}-2 x_{2} x_{3} h_{3}+x_{2} B_{2}+x_{3} B_{3}\right]\right. \\
& \left. \pm 2 x_{3}\left[2 x_{3} h_{1}+3 x_{2} h_{2}+\left(4 x_{1}-x_{3}^{2}\right) h_{3}+x_{2} C_{2}+x_{3} C_{3}\right]\right\} .
\end{aligned}
$$


Thus, we get $\bmod T Q S W L_{G_{0}}: x_{2}^{2} \equiv 0, x_{3}^{2} \equiv 0, x_{2} x_{3} \equiv 0, x_{1} x_{3} \equiv 0$ and $x_{1} x_{2} \equiv 0$. Hence, $\mathbf{C}_{x} / T Q S W L_{G_{0}} \simeq \mathbf{C}_{x_{1}}$. According to Remark 2.10, the germ $G$ is quasi swallowtail equivalent to $\pm x_{2}^{2} \pm x_{3}^{2}+\varphi_{1}\left(x_{1}\right)$ with $\varphi_{1} \in \mathcal{M}_{x_{1}}^{3}$.

Similarly, we can show that

- the germ $H= \pm x_{1}^{2} \pm x_{3}^{2}+h_{3}(x)$, where $h_{3} \in \mathcal{M}_{x}^{3}$ is quasi swallowtail equivalent to $\pm x_{1}^{2} \pm$ $x_{3}^{2}+\varphi_{2}\left(x_{2}\right)$ with $\varphi_{2} \in \mathcal{M}_{x_{2}}^{3}$, and

- the germ $P= \pm x_{1}^{2} \pm x_{2}^{2}+p_{3}(x)$, where $p_{3} \in \mathcal{M}_{x}^{3}$ is quasi swallowtail equivalent to $\pm x_{1}^{2} \pm x_{2}^{2}+$ $x_{1} \varphi_{3}\left(x_{3}\right)+\varphi_{4}\left(x_{3}\right)$, where $\varphi_{3} \in \mathcal{M}_{x_{3}}^{2}$ and $\varphi_{4} \in \mathcal{M}_{x_{3}}^{3}$.

Lemma 3.5 A function germ $f\left(x, y_{1}\right)= \pm x_{1}^{2} \pm x_{2}^{2} \pm x_{3}^{2}+f_{3}\left(x, y_{1}\right)$ with $f_{3} \in \mathcal{M}_{x, y_{1}}^{3}$, is quasi swallowtail equivalent to $\widetilde{f}\left(x, y_{1}\right)= \pm x_{1}^{2} \pm x_{2}^{2} \pm x_{3}^{2}+x_{1} \phi_{1}\left(y_{1}\right)+x_{2} \phi_{2}\left(y_{1}\right)+x_{3} \phi_{3}\left(y_{1}\right)+\phi_{4}\left(y_{1}\right)$ where $\phi_{1}, \phi_{2}, \phi_{3} \in \mathcal{M}_{y_{1}}^{2}$ and $\phi_{4} \in \mathcal{M}_{y_{1}}^{3}$.

Proof. Consider the principal part $f_{0}= \pm x_{1}^{2} \pm x_{2}^{2} \pm x_{3}^{2}$. Then, we get $\bmod T Q S W L_{f_{0}}: x_{1}^{2} \equiv 0$, $x_{2}^{2} \equiv 0, x_{3}^{2} \equiv 0, x_{1} x_{2} \equiv 0, x_{1} x_{3} \equiv 0$ and $x_{2} x_{3} \equiv 0$. Hence, we have

$\mathbf{C}_{x, y_{1}} / T Q S W L_{f_{0}} \simeq\left\{x_{1} \varphi_{1}\left(y_{1}\right)+x_{2} \varphi_{2}\left(y_{1}\right)+x_{3} \varphi_{3}\left(y_{1}\right)+\varphi_{4}\left(y_{1}\right): \varphi_{1}, \varphi_{2}, \varphi_{3}, \varphi_{4} \in \mathbf{C}_{y_{1}}\right\}$. Due to the constraint in the lemma on $f_{3}$, the claim of the lemma follows.

\subsection{Proof of the Main Theorem 3.1}

Lemmas 3.3, 3.4 and 3.5 yield that all simple quasi swallowtail singularities are among the following germs:

1. $G_{1}= \pm x_{2}^{2} \pm x_{3}^{2}+\varphi_{1}\left(x_{1}\right)$ where $\varphi_{1} \in \mathcal{M}_{x_{1}}^{3}$.

2. $G_{2}= \pm x_{1}^{2} \pm x_{3}^{2}+\varphi_{2}\left(x_{2}\right)$ where $\varphi_{2} \in \mathcal{M}_{x_{2}}^{3}$.

3. $G_{3}= \pm x_{1}^{2} \pm x_{2}^{2}+x_{1} \varphi_{3}\left(x_{3}\right)+\varphi_{4}\left(x_{3}\right)$ where $\varphi_{3} \in \mathcal{M}_{x_{3}}^{2}$ and $\varphi_{4} \in \mathcal{M}_{x_{3}}^{3}$.

4. $G_{4}= \pm x_{1}^{2} \pm x_{2}^{2} \pm x_{3}^{2}+x_{1} \phi_{1}\left(y_{1}\right)+x_{2} \phi_{2}\left(y_{1}\right)+x_{3} \phi_{3}\left(y_{1}\right)+\phi_{4}\left(y_{1}\right)$ where $\phi_{1}, \phi_{2}, \phi_{3} \in \mathcal{M}_{y_{1}}^{2}$ and $\phi_{4} \in \mathcal{M}_{y_{1}}^{3}$.

Using Lemma 2.9, one can easily prove the results below.

Consider the germ $G_{1}$. Let $\varphi_{1}\left(x_{1}\right)=a_{k} x_{1}^{k}+\widetilde{\varphi}_{1}\left(x_{1}\right)$, where $a_{k} \neq 0, k \geq 3$ and $\widetilde{\varphi}_{1} \in \mathcal{M}_{x_{1}}^{k+1}$. Then, $G_{1}$ is quasi swallowtail equivalent to the germ $\mathcal{Q}_{k}: \pm x_{2}^{2} \pm x_{3}^{2} \pm x_{1}^{k}$.

Similarly the germ $G_{2}$ can be reduced to one of the functions $\mathcal{T}_{k}: \pm x_{1}^{2} \pm x_{3}^{2} \pm x_{2}^{k}, k \geq 3$.

Next, consider the germ $G_{3}$. If $\varphi_{4}$ contains a term $a x_{3}^{3}, a \neq 0$, then $G_{3}$ is quasi swallowtail equivalent to $\mathcal{D}_{3}: \pm x_{1}^{2} \pm x_{2}^{2}+x_{3}^{3}$. Otherwise, in the most general case, $G_{2}$ is quasi swallowtail equivalent to a non-simple germ $\mathcal{D}_{4}: \pm x_{2}^{2} \pm\left(x_{1}+\delta x_{3}^{2}\right)^{2} \pm x_{3}^{4}, \delta \in \mathbb{R}$.

Finally, consider the germ $G_{4}= \pm x_{1}^{2} \pm x_{2}^{2} \pm x_{3}^{2}+x_{1}\left(a_{2} y_{1}^{2}+a_{3} y_{1}^{3}+a_{4} y_{1}^{4}+\ldots\right)+x_{2}\left(b_{2} y_{1}^{2}+b_{3} y_{1}^{3}+\right.$ $\left.b_{4} y_{1}^{4}+\ldots\right)+x_{3}\left(c_{2} y_{1}^{2}+c_{3} y_{1}^{3}+c_{4} y_{1}^{4}+\ldots\right)+d_{3} y_{1}^{3}+d_{4} y_{1}^{4}+d_{5} y_{1}^{5}+\ldots$

- If $d_{3} \neq 0$, then $G_{4}$ is quasi swallowtail equivalent to $\pm x_{1}^{2} \pm x_{2}^{2} \pm x_{3}^{2}+y_{1}^{3}$, which can be written equivalently as $\mathcal{N}_{2,2,2,3}: \pm\left(x_{1}+y_{1}^{2}\right)^{2} \pm\left(x_{2}+y_{1}^{2}\right)^{2} \pm\left(x_{3}+y_{1}^{2}\right)^{2}+y_{1}^{3}$.

- If $d_{3}=0, a_{2} \neq 0$ and $d_{4} \neq \pm \frac{a_{2}^{2}}{4}$, then $G_{4}$ is quasi swallowtail equivalent to $\pm x_{1}^{2} \pm x_{2}^{2} \pm x_{3}^{2}+x_{1} y_{1}^{2} \pm$ $y_{1}^{4}$, which is also quasi swallowtail equivalent to $\mathcal{N}_{2,2,2,4}: \pm\left(x_{1}+y_{1}^{2}\right)^{2} \pm\left(x_{2}+y_{1}^{2}\right)^{2} \pm\left(x_{2}+y_{1}^{2}\right)^{2} \pm y_{1}^{4}$. 
- If $d_{3}=0, a_{2} \neq 0$ and $d_{4}= \pm \frac{a_{2}^{2}}{4}$, then we get the classes $\mathcal{N}_{2,2,2, k}: \pm\left(x_{1}+y_{1}^{2}\right)^{2} \pm\left(x_{2}+y_{1}^{2}\right)^{2} \pm$ $\left(x_{2}+y_{1}^{2}\right)^{2} \pm y_{1}^{k}$, where $k \geq 5$

- If $d_{3}=a_{2}=0, b_{2} \neq 0$ and $d_{4} \neq \pm \frac{b_{2}^{2}}{4}$, then $G_{4}$ is quasi swallowtail equivalent to $\pm x_{1}^{2} \pm x_{2}^{2} \pm$ $x_{3}^{2} \pm x_{2} y_{1}^{2} \pm y_{1}^{4}$, which is equivalent to $\mathcal{N}_{3,2,2,4}: \pm\left(x_{1}+y_{1}^{3}\right)^{2} \pm\left(x_{2}+y_{1}^{2}\right)^{2} \pm\left(x_{3}+y_{1}^{2}\right)^{2} \pm y_{1}^{4}$.

- If $d_{3}=a_{2}=0, b_{2} \neq 0$ and $d_{4}= \pm \frac{b_{2}^{2}}{4}$, then we get a non-simple class $\mathcal{N}_{3,2,2,5}: \pm\left(x_{1}+y_{1}^{3}\right)^{2} \pm\left(x_{2}+y_{1}^{2}\right)^{2}+ \pm\left(x_{2}+y_{1}^{2}\right)^{2}+\alpha y_{1}^{5}$ with $\alpha \in \mathbb{R} \backslash\{0\}$.

- If $d_{3}=a_{2}=b_{2}=0$, and $c_{4} \neq 0$ and $d_{4} \neq \pm \frac{c_{2}^{2}}{4}$, then we get the class $\pm x_{1}^{2} \pm x_{2}^{2} \pm x_{3}^{2} \pm x_{3} y_{1}^{2} \pm y_{1}^{4}$, or, equivalently, the $\mathcal{N}_{3,3,2,4}$ class: $\pm\left(x_{1}+y_{1}^{3}\right)^{2} \pm\left(x_{2}+y_{1}^{3}\right)^{2} \pm\left(x_{3}+y_{1}^{2}\right)^{2} \pm y_{1}^{4}$.

- If $d_{3}=a_{2}=b_{2}=0, c_{2} \neq 0$ and $d_{4}= \pm \frac{c_{2}^{2}}{4}$, then we get a non-simple class $\mathcal{N}_{3,3,2,5}: \pm\left(x_{1}+y_{1}^{3}\right)^{2} \pm\left(x_{2}+y_{1}^{3}\right)^{2} \pm\left(x_{2}+y_{1}^{2}\right)^{2}+\gamma y_{1}^{5}$ with $\gamma \in \mathbb{R} \backslash\{0\}$.

- If $d_{3}=a_{2}=b_{2}=c_{2}=0$ and $d_{4} \neq 0$, then we get the class $\pm x_{1}^{2} \pm x_{2}^{2} \pm x_{3}^{2} \pm y_{1}^{4}$ or, equivalently, the $\mathcal{N}_{3,3,3,4}$ class: $\pm\left(x_{1}+y_{1}^{3}\right)^{2} \pm\left(x_{2}+y_{1}^{3}\right)^{2} \pm\left(x_{3}+y_{1}^{3}\right)^{2} \pm y_{1}^{4}$.

This finishes the proof of the theorem.

\section{BIFURCATION DIAGRAMS AND CAUSTICS OF SIMPLE QUASI SWAL- LOWTAIL SINGULARITIES}

A quasi swallowtail miniversal deformation of a function germ $f:\left(\mathbb{R}^{n}, 0\right) \rightarrow(\mathbb{R}, 0)$ may be constructed in the standard way as

$$
F(x, y, \lambda)=f(x, y)+\sum_{i=0}^{\tau-1} \lambda_{i} e_{i}(x, y)
$$

where $e_{0}, \ldots, e_{\tau-1} \in \mathbf{C}_{x, y}$ project to a basis of $\mathbf{C}_{x, y} / T Q S W L_{f}$. We will use the notation $F_{\lambda}$ for $\left.F\right|_{\lambda=\text { const }}$, so that $F_{0}=f$.

Definition 4.1 The quasi swallowtail bifurcation diagram of a function germ $f$ is the set of all points $\lambda$ in the base $\mathbb{R}^{\tau}$ of its quasi swallowtail miniversal deformation for which

- either the set $\left\{F_{\lambda}=0\right\} \subset \mathbb{R}^{n}$ is singular,

- or a singularity of $\left\{F_{\lambda}=0\right\}$ is on $\Gamma_{s w l}$.

Respectively, this diagram consists of two components, $W_{1}$ and $W_{2}: W_{2} \subset W_{1}, \operatorname{dim} W_{j}=\tau-j$.

Now assume that $e_{0}=1$ in (4), and all the other $e_{i}$ are from $\mathcal{M}_{x, y}$. Following the standard approach, we call the space $\mathbb{R}^{\tau-1}$ of the parameter $\lambda_{1}, \ldots, \lambda_{\tau-1}$ the base of a truncated quasi swallowtail miniversal deformation of $f$.

Definition 4.2 Consider the projection map $\Pi: \mathbb{R}^{\tau} \rightarrow \mathbb{R}^{\tau-1}$ between the two bases, forgetting $\lambda_{0}$. The quasi swallowtail caustic of a function $f$ is a hypersurface in the base $\mathbb{R}^{\tau-1}$ which is a union of the $\Pi$-image $\Sigma_{1}$ of the singular points of the set $W_{1} \subset \mathbb{R}^{\tau}$, and of the set $\Sigma_{2}=\Pi\left(W_{2}\right)$.

Remark 4.3 In terms of Section 5 below, the component $W_{1}$ is the critical value set of the Lagrangian map of the manifold $L$, and $W_{2}$ is the image of the border $\Gamma_{s w l}$. 
The versal deformations listed below provide an explicit description of the bifurcation diagrams and caustics of simple quasi swallowtail singularities.

Proposition 4.4 Quasi swallowtail miniversal deformations of simple quasi swallowtail classes are as follows:

\begin{tabular}{|c|l|c|}
\hline Singularity & \multicolumn{1}{|c|}{ Miniversal deformation } & Restrictions \\
\hline $\mathcal{Q}_{k}$ & $\pm x_{2}^{2} \pm x_{3}^{2} \pm x_{1}^{k}+\sum_{i=0}^{k-1} \lambda_{i} x_{1}^{i}+\lambda_{k} x_{2}+\lambda_{k+1} x_{3}$ & $k \geq 2$ \\
\hline $\mathcal{T}_{k}$ & $\pm x_{1}^{2} \pm x_{3}^{2} \pm x_{2}^{k}+\sum_{i=0}^{k-1} \lambda_{i} x_{2}^{i}+\lambda_{k} x_{1}+\lambda_{k+1} x_{1}+\lambda_{k+2} x_{1} x_{2}$ & $k \geq 3$ \\
\hline $\mathcal{D}_{3}$ & $\pm x_{1}^{2} \pm x_{2}^{2} \pm x_{3}^{3}+\lambda_{0}+\lambda_{1} x_{1}+\lambda_{2} x_{2}+\lambda_{3} x_{3}+\lambda_{4} x_{3}^{2}+\lambda_{5} x_{1} x_{3}$ & \\
& $+\lambda_{6} x_{2} x_{3}$ & \\
\hline $\mathcal{N}_{2,2,2, k}$ & $\pm\left(x_{1}+y_{1}^{2}\right)^{2} \pm\left(x_{2}+y_{1}^{2}\right)^{2} \pm\left(x_{3}+y_{1}^{2}\right)^{2} \pm y_{1}^{k}+\sum_{i=0}^{k-2} \lambda_{i} y_{1}^{i}$ & $k \geq 3$ \\
& $+\lambda_{k-1} x_{1}+\lambda_{k} x_{2}+\lambda_{k+1} x_{3}+\lambda_{k+2} x_{1} y_{1}+\lambda_{k+3} x_{2} y_{1}+\lambda_{k+4} x_{3} y_{1} \cdot$ & \\
\hline $\mathcal{N}_{3,2,2,4}$ & $\pm\left(x_{1}+y_{1}^{3}\right)^{2} \pm\left(x_{2}+y_{1}^{2}\right)^{2} \pm\left(x_{3}+y_{1}^{2}\right)^{2} \pm y_{1}^{4}+\lambda_{0}+\lambda_{1} y_{1}+\lambda_{2} y_{1}^{2}$ & \\
& $+\lambda_{3} x_{1}+\lambda_{4} x_{2}+\lambda_{5} x_{3}+\lambda_{6} x_{1} y_{1}+\lambda_{7} x_{2} y_{1}+\lambda_{8} x_{3} y_{1}+\lambda_{9} x_{1} y_{1}^{2}$. & \\
\hline $\mathcal{N}_{3,3,2,4}$ & $\pm\left(x_{1}+y_{1}^{3}\right)^{2} \pm\left(x_{2}+y_{1}^{3}\right)^{2} \pm\left(x_{3}+y_{1}^{2}\right)^{2} \pm y_{1}^{4}+\lambda_{0}+\lambda_{1} y_{1}+\lambda_{2} y_{1}^{2}$ & \\
& $+\lambda_{3} x_{1}+\lambda_{4} x_{2}+\lambda_{5} x_{3}+\lambda_{6} x_{1} y_{1}+\lambda_{7} x_{2} y_{1}+\lambda_{8} x_{3} y_{1}+\lambda_{9} x_{1} y_{1}^{2}$ & \\
& $+\lambda_{10} x_{2} y_{1}^{2}$. & \\
\hline $\mathcal{N}_{3,3,3,4}$ & $\pm\left(x_{1}+y_{1}^{3}\right)^{2} \pm\left(x_{2}+y_{1}^{3}\right)^{2} \pm\left(x_{3}+y_{1}^{3}\right)^{2} \pm y_{1}^{4} \lambda_{0}+\lambda_{1} y_{1}+\lambda_{2} y_{1}^{2}$ & \\
& $+\lambda_{3} x_{1}+\lambda_{4} x_{2}+\lambda_{5} x_{3}+\lambda_{6} x_{1} y_{1}+\lambda_{7} x_{2} y_{1}+\lambda_{8} x_{3} y_{1}+\lambda_{9} x_{1} y_{1}^{2}$ & \\
$+\lambda_{10} x_{2} y_{1}^{2}++\lambda_{11} x_{3} y_{1}^{2}$. & \\
\hline
\end{tabular}

All simple quasi swallowtail singularities are the $A_{k}$ singularities with respect to the standard right equivalence. So, the first component of the quasi swallowtail bifurcation diagram of a simple quasi swallowtail function is a product of a generalized swallowtail and $\mathbb{R}^{\tau-k}$. A similar observation is valid for the first components of the caustics. In particular, the bifurcation diagram of $\mathcal{Q}_{2}$ in $\mathbb{R}^{4}$ is a smooth hypersurface and a swallowtail on it. The $\mathcal{Q}_{2}$ caustic in $\mathbb{R}^{3}$ is a union of a smooth surface and a swallowtail.

\section{APPLICATION TO LAGRANGIAN BORDER SINGULARITIES}

Standard notions and basic definitions concerning Lagrangian singularities can be found in [8].

Singularities of Lagrangian projections (mappings) are essentially the singularities of their generating families treated as families of functions depending on parameters and considered up to the right equivalence depending on parameters and addition of functions in parameters. In particular, the caustic $\Sigma(L)$ of a Lagrangian projection of a Lagrangian submanifold $L$ coincides with the set of values of the parameters $\lambda$ of the generating family $F(w, \lambda)$ for which the family member $F_{\lambda}$ has a non-Morse critical point. 
Stability of a Lagrangian projection with respect to symplectomorphisms preserving the fibration structure corresponds to the versality of the generating family with respect to the $\mathcal{R}_{+}$-equivalence group.

Consider the standard symplectic space $M=T^{*} \mathbb{R}^{n}$ with coordinates $q$ on the base $\mathbb{R}^{n}$ and dual coordinates $p$ on the fibers of the Lagrangian projection $\pi: T^{*} \mathbb{R}^{n} \rightarrow \mathbb{R}^{n}$.

Locally any Lagrangian submanifold $L^{n}$ in an ambient symplectic space $M$ is determined by a generating family of functions $F(w, q)$ in variables $w \in \mathbb{R}^{m}$ and parameters $q \in \mathbb{R}^{n}$ according to the standard formula:

$$
L=\left\{(p, q) \in \mathbb{R}^{n} \times \mathbb{R}^{n}: \exists w \in \mathbb{R}^{m}, \frac{\partial F}{\partial w_{i}}=0, p=\frac{\partial F}{\partial q}\right\},
$$

provided that the Morse non-degeneracy condition [ the matrix $\left(\begin{array}{ll}\frac{\partial^{2} F}{\partial w_{i} \partial w_{j}} & \frac{\partial^{2} F}{\partial w_{i} \partial q_{j}}\end{array}\right)$ has rank $\left.n\right]$ holds. The condition guarantees $L$ being a smooth manifold.

Definition 5.1 [8] Two family germs $F_{i}(w, q), w \in \mathbb{R}^{m}, q \in \mathbb{R}^{n}, i=1,2$, at the origin are called $\mathcal{R}_{+}$-equivalent if there exists a diffeomorphism $\Phi:(w, q) \mapsto(W(w, q), Q(q))$ and a smooth function $\Theta$ of the parameters $q$ such that $F_{2}(w, q)=\left(F_{1} \circ \Phi\right)(w, q)+\Theta(q)$.

Following applications of quasi boundary, quasi corner and quasi cusp equivalence relations considered in $[2,3,4]$, we introduce

Definition $5.2[4]$ A pair $(L, \Gamma)$ consisting of a Lagrangian submanifold $L^{n}$ in an ambient symplectic space $M$ and an $(n-1)$-dimensional isotropic variety $\Gamma \subset L$ is called a Lagrangian submanifold with a border $\Gamma$.

Definition 5.3 [4] Lagrangian projections of two Lagrangian submanifolds with borders $\left(L_{i}, \Gamma_{i}\right)$, $i=1,2$, are Lagrange equivalent if there exists a symplectomorphism of the ambient space $M$ which preserves the $\pi$-bundle structure and sends one pair to the other.

The notions of stability and simplicity of Lagrangian submanifolds with borders with respect to this Lagrangian equivalence are straightforward.

Up to a Lagrange equivalence we may assume that in a vicinity of a base point the tangent space to $L$ has a regular projection onto the fiber of $\pi$ and the coordinates $p$ can be taken as coordinates $w$ on the fibers of the source space of the generating family.

Generating family is defined up to $\mathcal{R}_{+}$-equivalence. So having two Lagrange equivalent pairs $\left(L_{i}, \Gamma_{i}\right)$ we can choose a generating family for one of them in coordinates $p, q$ and the generating family for the second pair in transformed coordinates $\widetilde{P}(p)$ so that the projection of $\Gamma_{1}$ to $p$ coordinate subspace coincides with the projection of $\Gamma_{2}$ to the $\widetilde{P}$-coordinate subspace.

Assume that the $\Gamma_{i}$ are borders, $i=1,2$. Rename the coordinates $p$ by $w$ and $q$ by $\lambda$. Let $g_{i}(w)=0$ be the equation of the border $\Gamma_{i}, i=1,2$.

Now we have generating families $F_{i}(w, \lambda)$ for both submanifolds such that the critical points of $F_{i}$ with respect to variables $w$ at the set $\left\{g_{i}(w)=0\right\}$ correspond to the border $\Gamma_{i}$.

Hence, the Lagrange equivalence of pairs $\left(L_{i}, \Gamma_{i}\right), \quad i=1,2$, gives rise to an equivalence of the generating families $F_{i}$ which is a pseudo border equivalence and addition with a function in parameters.

Moreover the following holds. 
Proposition 5.4 Let $\left(L_{t}, \Gamma_{t}\right), t \in[0,1]$, be a family of equivalent pairs of Lagrangian submanifolds with a cylinder over a swallowtail stratum. Then the respective generating families are quasi swallowtail equivalent up to addition of functions depending on parameters.

The above equivalence of generating families will be called the quasi swallowtail +-equivalence.

The last proposition and the classification of simple quasi swallowtail singularities imply the following theorem.

Theorem 5.5 1. A germ $(L, \Gamma)$ is stable if and only if its arbitrary generating family is quasi border +-versal, that is, versal with respect to the quasi border equivalence and addition of functions in parameters.

2. Any stable and simple projection of a Lagrangian submanifold with a cylinder over a swallowtail stratum is symplectically equivalent to the projection determined by a generating family which is a quasi swallowtail +-versal deformation of one of the classes from Theorem 3.1.

Proof. Suppose that a germ $\left(L_{0}, \Gamma_{0}\right)$ is stable. Then any germ $(\widetilde{L}, \widetilde{\Gamma})$ close $\left(L_{0}, \Gamma_{0}\right)$ is Lagrange equivalent to it.

Assume we have a family $\left(L_{t}, \Gamma_{t}\right)$ of deformations of $\left(L_{0}, \Gamma_{0}\right)$, with $t \in[0,1]$. Also assume that there is a family of diffeomorphism $\theta_{t}: T^{*} \mathbb{R}^{n} \rightarrow T^{*} \mathbb{R}^{n}$ which preserves Lagrange fibration $\pi: T^{*} \mathbb{R}^{n} \rightarrow \mathbb{R}^{n},(p, q) \mapsto q$ and the standard symplectic form $\omega$, and maps $\left(L_{t}, \Gamma_{t}\right)$ to $\left(L_{0}, \Gamma_{0}\right)$.

Consider families depending on $t$ of respective generating families $G_{t}(w, q)$ of $\left(L_{t}, \Gamma_{t}\right)$ with $t \in$ $[0,1]$ and $G_{0}$ being a generating family of the pair $\left(L_{0}, \Gamma_{0}\right)$. By Proposition 5.4, all the $G_{t}$ are quasi swallowtail +-equivalent. Thus, there exist a family of diffeomorphisms $\Phi_{t}:(w, q) \mapsto\left(\widetilde{w}_{t}(w, q), Q_{t}(q)\right)$ and a family $\Psi_{t}$ of smooth functions of the parameters $q$ such that: $G_{t} \circ \Phi_{t}=G_{0}+\Psi_{t}$, and the critical points sets $\left\{\frac{\partial G_{t}}{\partial w}=0\right\}$ correspond to the Lagrangian submanifolds $L_{t}$. This yields, in particular, that $G_{0}$ is versal with respect to quasi border +-equivalence.

By reversing the previous argument we prove the converse claim.

The second part of the theorem is a consequence of the classification of function germs with respect to the quasi swallowtail equivalence.

\section{CONCLUSION}

The further study of similar non-standard equivalence relation and its comparison with standard one will give extra information on the nature of singularity classes in various optimization problems and problems in variations theory with constraints, on the adjacencies of respective singularity classes and on their invariants.

ACKNOWLEDGEMENTS: I am deeply grateful to Vladimir Zakalyukin who introduced me to the idea of quasi border equivalence relation. I am also thankful to Victor Goryunov for a helpful discussion and his highly useful comments on the first draft of the paper. 


\section{References}

[1] V.M. Zakalyukin, Quasi-projections. Proceedings of the Steklov Institute of Mathematics 259 (2007), no. 1, 273-280.

[2] V.M. Zakalyukin, Quasi singularities. Geometry and topology of caustics - CAUSTICS '06, Banach Center Publications 82 (2008), Polish Acad. Sci. Inst. Math., Warsaw, 215-225.

[3] F.D. Alharbi and V.M. Zakalyukin, Quasi corner singularities. Proceedings of the Steklov Institute of Mathematics 270 (2010), no. 1, 1-14.

[4] F.D. Alharbi, Quasi cusp singularities. The Journal of Singularities, volume 12(2015), 1-18.

[5] V.I Arnold, Singularities of caustics and wave fronts. Mathematics and its Applications 62, Kluwer Academic Publishers, Dordrecht, 1990.

[6] F.D. Alharbi, Bifurcation diagrams and caustics of simple quasi border singularities. Topology and its Applications 159 (2011), no. 2, 381-388.

[7] V. M. Zakalyukin, Reconstructions of wave fronts depending on one parameter, Functional Analysis and its Applications 10 (1976), 139140.

[8] V.I. Arnold, S.M. Gusein-Zade and A.N. Varchenko, Singularities of differentiable maps. Vol. I. Monographs in Mathematics 82, Birkhäuser Boston, Boston, 1985.

\section{AUTHOR'S BIOGRAPHY:}

Full Name: Dr. Fawaz Deghaileeb Alharbi. Title: Assistant Professor of Mathematics.

Institution: Umm Alqura University, Makkah, Saudi Arabia. Nationality: Saudi.

Date and Place of Birth: 7/1/1977, Makkah, Saudi Arabia. Marital Status: Married.

Qualifications: B.Sc.(Mathematics-Umm Alqura University, M.Sc.and Ph.D.(Mathematics-Liverpool University, U.K). Research Interests: Singulariy Theory, symplectic geometry.

\section{Professional experience:}

1. Worked as a teacher at Minsitary of Education from 7th of Sep. 1999 to 28th Nov. 2002.

2. Served as a demonstrator at Umm Alqura University, department of Mathematics from 29th of Nov.2002 until 26th Dec.2011.

3. Working as an assistant professor from 27th Dec.2011 until now.

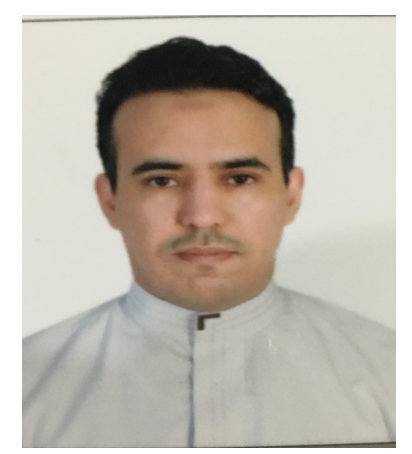

